\title{
Prevalence and Characteristics of Neuropathy in Carcinoma Colon Patients on FOLFOX4 Therapy
}

\author{
Aman Bharti, Isha Arora ${ }^{2}$,Vikas Chalotra ${ }^{3}$ \\ ${ }^{1}$ Assistant Professor, Department of General Medicine, Guru Gobind Singh Medical College \&Hospital Faridkot, Punjab $151203,{ }^{2}$ Assistant Professor, \\ Department of General Medicine, VIMS Gajraula, UP, ${ }^{3}$ Assistant Professor, Department of General Surgery, Guru Gobind Singh Medical College \& \\ Hospital Faridkot, Punjab 151203.
}

\section{Abstract}

Background: Colorectal cancer (CRC) is the third most common cancer in men and the second in women worldwide. The burden of colorectal cancer has risen rapidly in economically developed asian countries.Radical surgical resection is the main treatment of colorectal carcinoma. FOLFOX 4 is best first line treatment regime for colorectal carcinoma. Peripheral neuropathy is the important side effect of FOLFOX 4 regime. The aim of this study is to study the prevalence and characteristics of neuropathy in carcinoma colon patients on FOLFOX 4 therapy. Subjects and Methods: 60 patients of colorectal carcinoma on FOLFOX 4 therapy were included in the study. Out of 60 , 46 patients agreed for participation in the study after taking informed consent.All patients were questioned about symptoms General physical examination was carried out along with detailed neurological examination. Neurological examination also included modified total neuropathy score. Analysis was done on basis of symptoms, signs and modified total neuropathy score. Nerve conduction velocity study (total neuropathy score) was used only if the patient was willing for the test. Analysis was done at start of FOLFOX 4 chemotherapy, after 6 cycles and 12 cycles respectfully. Results: Among the 46 cases 25 were males (54.34\%) and 21 were females (45.65\%). Of a total of forty six patients peripheral neuropathy was diagnosed in twenty six patients based on modified neuropathy score hence the prevalence of neuropathy was found to be $56.52 \%$. Amongst motor symptoms, symptoms related to hand dexterity were commonly observed ( $\mathrm{n}=4,15.38 \%)$ followed by foot walking $(n=2,7.68 \%)$. Conclusion: Neuropathy was more common in females. Sensory neuropathy was more than mixed and motor neuropathy.Tingling was most common. Most patients had mild grade of neuropathy.

Keywords: Colorectal cancer, FOLFOX 4, Neuropathy,

Corresponding Author: Dr. Vikas Chalotra, Assistant Professor, Department of General Surgery, Guru Gobind Singh Medical College \& Hospital Faridkot, Punjab 151203

Received: October 2019

Accepted: October 2019

\section{Introduction}

Colorectal cancer $(\mathrm{CRC})$ is the third most common cancer in men and the second in women worldwide. ${ }^{[1]}$ Treatment of colorectal cancer depends on stage of cancer. Surgery is the treatment of choice for most patients with colorectal cancer. 5-FU has remained the mainstay of systemic chemotherapy. Various regimens combine 5-FU with high dose leucovorin (tetrahydofolate), because leucovorin potentiates the binding of 5-FU to thymidylatesynthetase, and the combination is more effective than 5-FU alone.Oxaliplatin is a diaminocyclohexane platinum that unlike other platinum compounds does not cause nephrotoxicity and has activity against CRC.FOLFOX 4 is recommended 2 weekly for 12 cycles. Various reported side effects of FOLFOX 4 therapy are ototoxicity, vestibular toxicity, anemia, neutropenia, leukopenia, thrombocytopenia, nausea, neuropathy. The most frequent dose limiting toxicity of Oxaliplatin is peripheral neuropathy (OXALIPN) next to Neutropenia. ${ }^{[2]}$ Oxaliplatin induced neurotoxicity has two distinct subtypes: acute and cumulative. ${ }^{[3,4]}$ Hereditary factors are believed to contribute to $20-30 \%$ of colorectal cancer patients. Diet rich in fats and red meat is associated with increased risk and inverse relationship is seen with development of colorectal carcinoma with vegetables and fruits consumption.Prolonged use of Non-Steroidal Anti-Inflammatory (NSAIDS), including aspirin, have been consistently associated with a reduced risk of colorectal cancer. Individuals with high levels of activity throughout their lives were found to have the lowest risk, diet rich in fiber is associated reduced risk. ${ }^{[5,6]}$

The standard chemotherapeutic regimen for colorectal carcinoma is 5-FU in combination with leucovorin whereas incorporation of oxaliplatin to this regimen enhances the therapeutic efficacy especially for metastatic colorectal carcinoma. The addition of oxaliplatin to 5FU/LV has doubled the response rate and increased the progression free survival in patients of metastatic disease. FOLFOX 4 (oxaliplatin/5-FU/LV regimen) is concluded to be the best first line treatment regimen for metastatic colorectal carcinoma. Chemotherapy-induced peripheral neuropathy (CIPN) describes damage to the peripheral nervous system caused by some chemotherapy agent. Some drugs may cause 
symptoms during or immediately after the first dose. The platinum compounds have been reported to have a delayed onset of symptoms, up to several weeks after the last dose. OXALIPN may be associated with varying combinations of weakness, autonomic changes, and sensory changes. Mild to moderate numbness and tingling of hands and feet can occur after 4-6 months of oxaliplatin therapy and may even develop 3-8 weeks after last dose. Symptoms can become severe with high cumulative dose. The acute, transient neurotoxicity observed with oxaliplatin occurs in nearly all patients. It is rapid in onset, occurring during or within hours of infusion. The symptoms are often induced or aggravated by exposure to cold. The sensory component consists of paresthesias and/or dysesthesias in the distal extremities and/or the perioral region. About $1 \%-2 \%$ of patients will report a transient cold-induced pharyngolaryngealdysesthesia, causing a feeling of difficulty in breathing. These sensory symptoms are less frequently paralleled by motor symptoms including: tetanic spasms, fasiculations, and prolonged muscular contractions. The dose-limiting, cumulative sensory neurotoxicity is seen in $10 \%-15 \%$ of patients after cumulative doses of 780-850 $\mathrm{mg} / \mathrm{m} 2$. Symptoms generally persist between cycles and increase in intensity with cumulative dose. Impaired sensation, sensory ataxia, and/ or deficit in fine sensorymotor coordination may ultimately occur. These symptoms are consistently reversible with the majority of patients recovering from grade 3 neurotoxicity to grade 1 or less within 6-12 months of therapy discontinuation. Lhermitte's sign, also referred to as the barber shop sign, is described as electric shock type sensation induced by forward flexion of the head. This occurred in patients who had received high cumulative doses of oxaliplatin $(>1000 \mathrm{mg} / \mathrm{m} 2) .{ }^{[7]}$

\section{Aims and Objectives}

To study the prevalence of neuropathy in carcinoma colon patients on FOLFOX 4 therapy.
To study the characteristics of neuropathy in carcinoma colon patients on FOLFOX 4 therapy.

\section{Subjects and Methods}

\section{Inclusion Criteria:}

All carcinoma colon cases on FOLFOX 4 therapy

\section{Exclusion criteria:}

(a) Presence of co-morbid neurological diseases and psychiatric diseases.

(b) Concurrent medical illness ( like diabetes mellitus, severe cardiopulmonary disease).

(c) Inability to give informed consent

All patients were questioned about symptoms like pain, numbness, tingling, burning or loss of sensation, weakness or heaviness of the hands and/or feet, blurred vision, reduced sweating, dizziness, bowel/bladder dysfunction and sexual dysfunction. General physical examination was carried out along with detailed neurological examination. Neurological examination also included modified total neuropathy score. Analysis was done on basis of symptoms, signs and modified total neuropathy score. Nerve conduction velocity study (total neuropathy score) was used only if the patient was willing for the test. Analysis was done at start of FOLFOX 4 chemotherapy, after 6 cycles and 12 cycles respectfully.

\section{Total Neuropathy Score (0-32 Points)}

A composite score combining subjective sensory symptoms, subjective report of symptoms and amount of difficulty with daily activities, deep tendonreflexes, manual muscle testing of distal muscles, pin sensibility, ability to feel vibration, and nerve conduction studies of sural and peroneal.

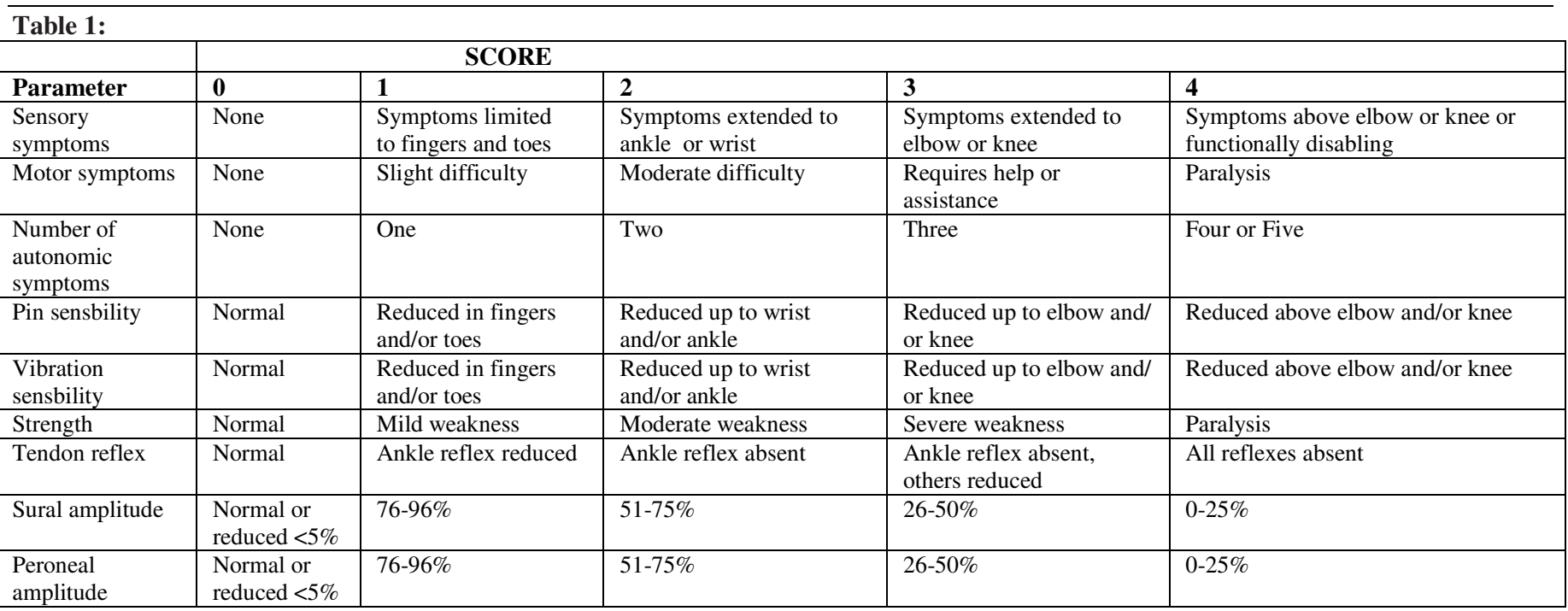

Reliability: Interrater-0.966, Intrarater-0.98613; Criterion Validity correlates with diabetic neuropathy gold standard measures ( $\mathrm{r}=0.71$ to 0.89 )

A modified version of the TNS (mTNS) excludes nerve conduction testing hence composite modified total neuropathy score is $0-24$.

Total neuropathy score:

Final diagnosis was made on basis of clinical history, neurological examination,modified total neuropathy score. 
Further evaluation was as follows:

(a) No neuropathy (no neurologic symptoms/ signs or test results or zero neuropathy score)

(b) Unequivocal neuropathy (Neuropathy score $\geq 1$, severe symptoms, gross neurological findings and marked test abnormalities)

Patients diagnosed with neuropathy were graded as mild (TNS 1 to 9), moderate (TNS 10-19), severe (TNS $\geq 20$ ), and underwent nerve conduction velocity study if possible. Analysis was done at start of chemotherapy, after 6 cycles and 12 cycles respectfully.

\section{Results}

Of a total of sixty patients, forty six patients with colorectal carcinoma on FOLFOX therapy agreed for participation in the study.

\section{Base Line Characteristics}

Among the 46 cases 25 were males (54.34\%) and 21 were females $(45.65 \%)$. Majority of patients were in age group of $30-60$ years. There were $5(10.86 \%)$ patients below 30 years age group and $9(19.56 \%)$ patients over age of sixty years. The mean age of patients was $48.97 \pm 14.77$ with a range of 14-76 years.

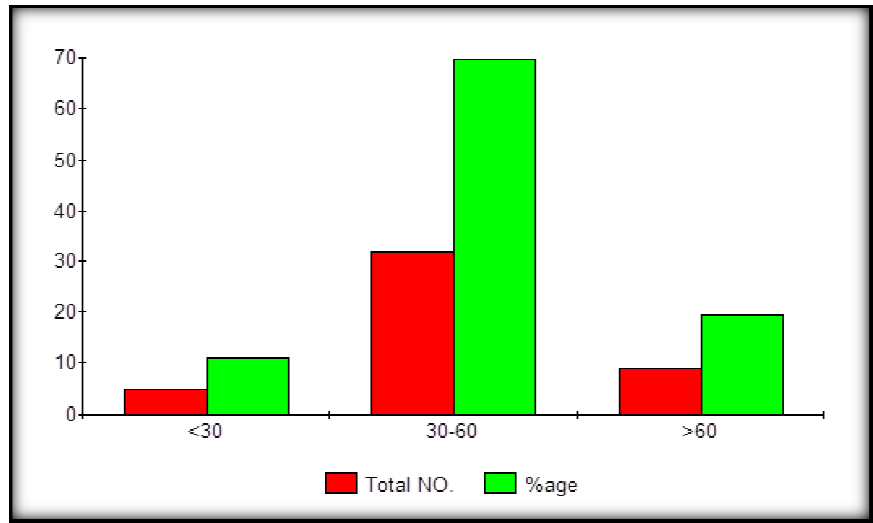

Figure 1: Age wise distribution of patients

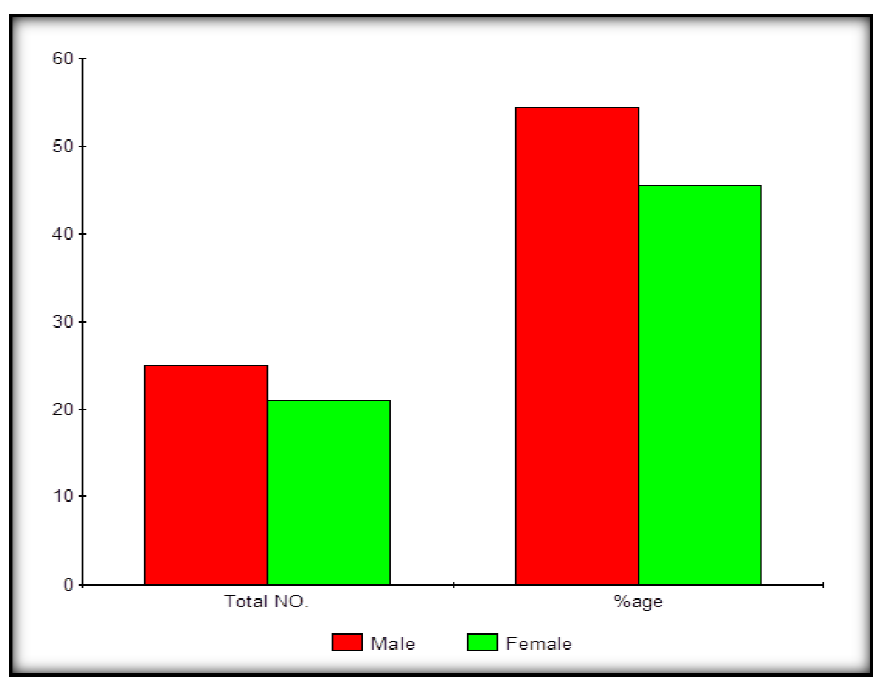

Figure 2: Gender wise distribution of patients

\section{Prevalence and Spectrum of Neuropathy}

Of a total of forty six patients peripheral neuropathy was diagnosed in twenty six patients based on modified neuropathy score hence the prevalence of neuropathy was found to be $56.52 \%$. Out of these 11 were males $(42.3 \%)$ and 15 were females $(7.69 \%)$. The occurrence of neuropathy was more in females $(15 / 21 ; 71.42 \%)$ as compared to males $(11 / 25 ; 44 \%)$. Majority of the patients developing neuropathy belonged to age group of 30-60 years $(n=16,61.53 \%)$. Twenty one patients had sensory and five patients had mixed neuropathy.

Table 2: Spectrum of Neuropathy

\begin{tabular}{|l|l|l|}
\hline Age(years) & Total No. & \%age \\
\hline$<30$ & 2 & 7.69 \\
\hline $30-60$ & 16 & 61.53 \\
\hline$>60$ & 8 & 30.76 \\
\hline Mean $( \pm$ SD) & $51.42( \pm 13.21)$ & \\
\hline Range & $21-76$ & \\
\hline Gender & & \\
\hline Male & 11 & 42.30 \\
\hline Female & 15 & 57.69 \\
\hline Neuropathy & & \\
\hline Sensory & 21 & 80.77 \\
\hline Motor & 0 & \\
\hline Mixed & 05 & 19.23 \\
\hline
\end{tabular}

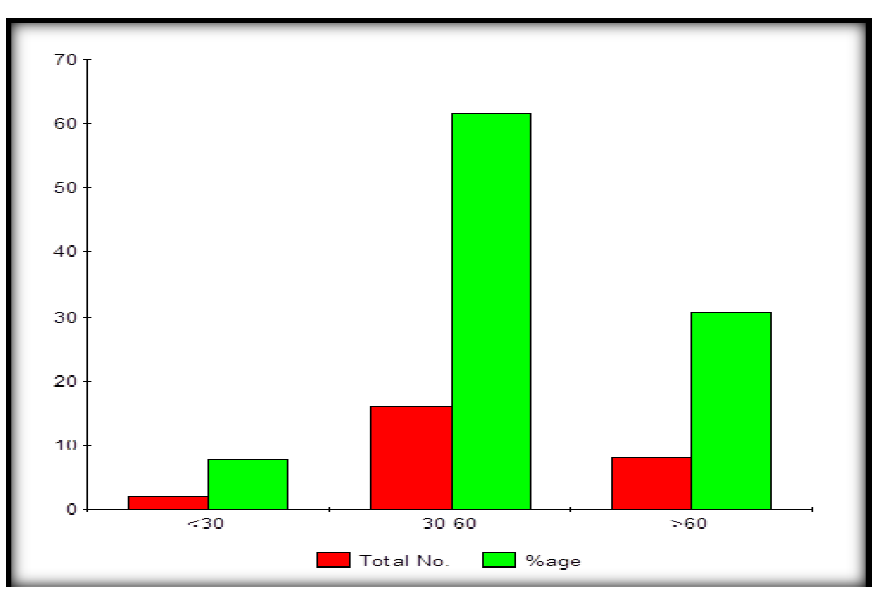

Figure 3: Age wise distribution of neuropathy

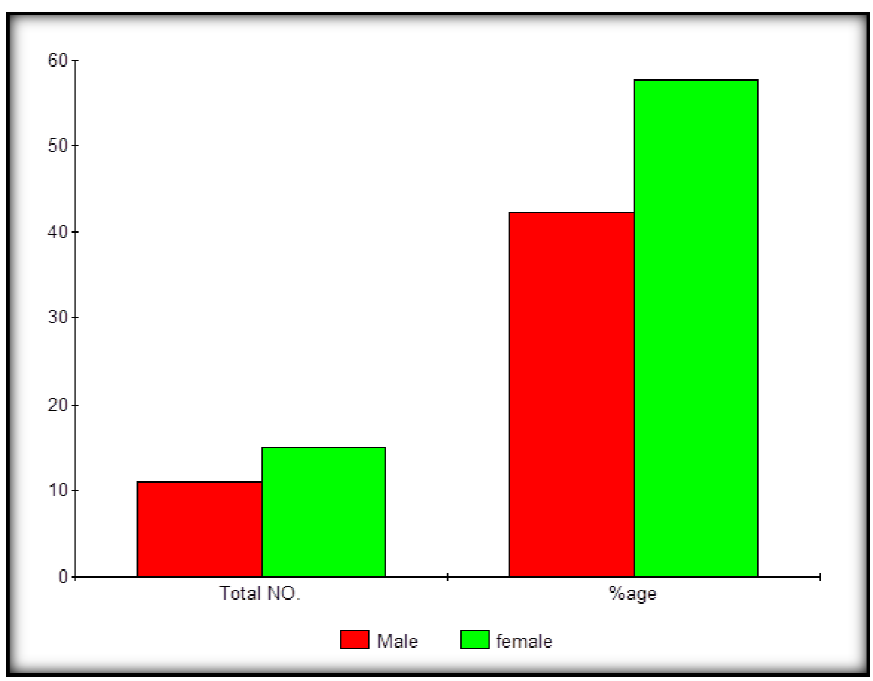

Figure 4: Gender wise distribution of neuropathy. 


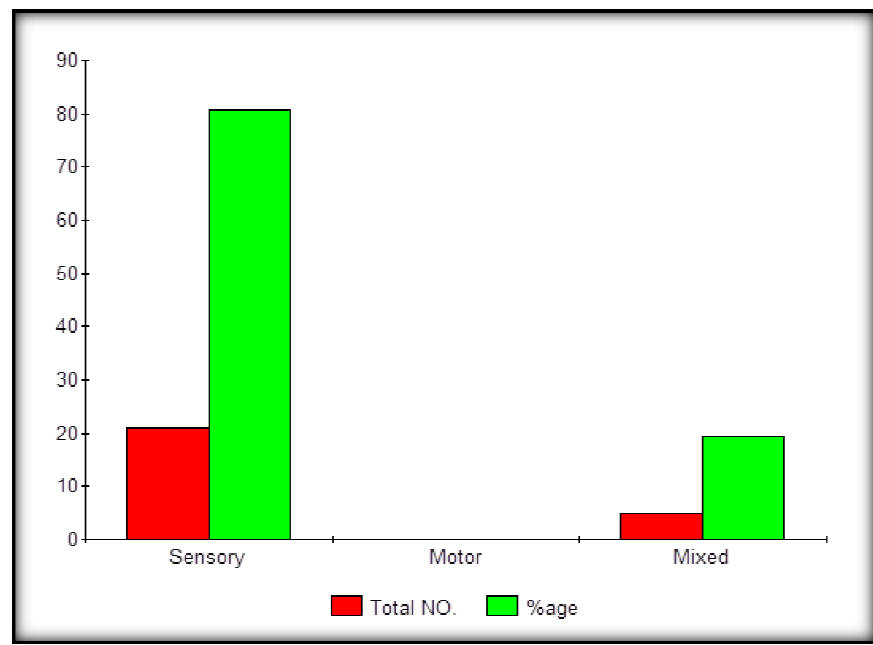

Figure 5: Pattern wise distribution of neuropathy.

\section{Sensory and Motor Symptoms}

Amongst sensory symptoms, tingling (paresthesias) was the commonest neuropathic symptom $(n=18,69.23 \%)$, followed by numbness $(n=5,19.23 \%)$. Neuropathic pain occurred in one patient (3.84\%). Amongst motor symptoms, symptoms related to hand dexterity were commonly observed $(n=4$, $15.38 \%)$ followed by foot walking $(\mathrm{n}=2,7.68 \%)$.

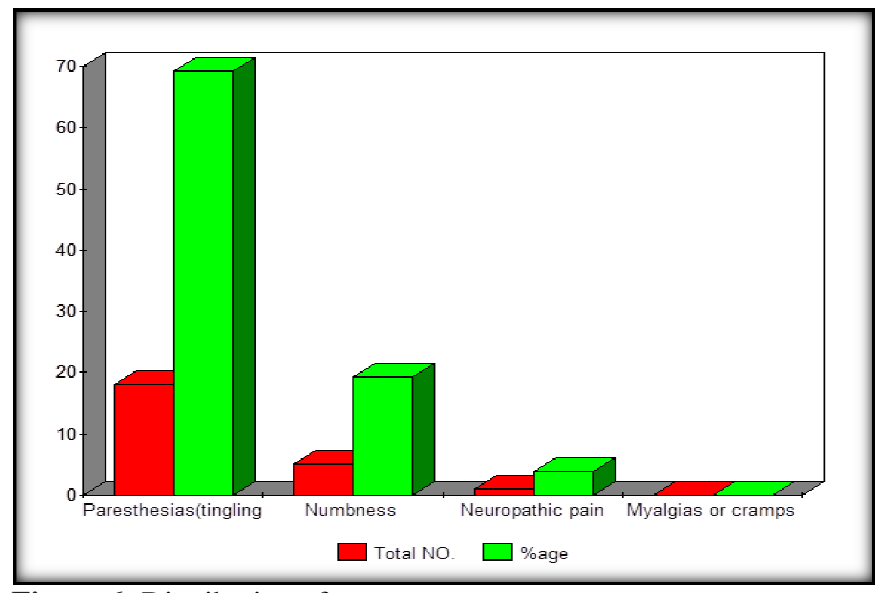

Figure 6: Distribution of sensory symptoms.

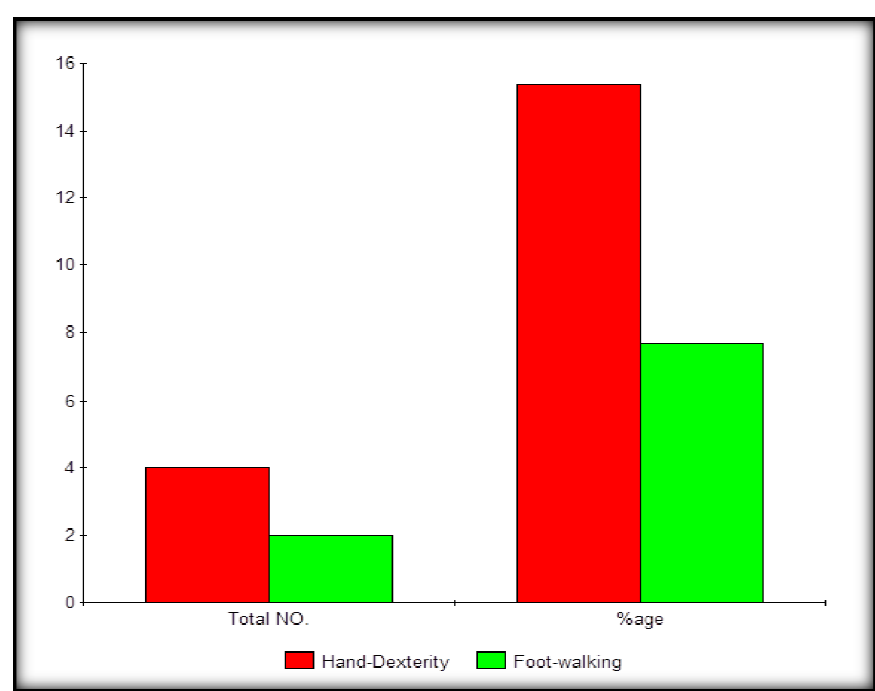

Figure 7: Distribution of motor symptoms.

\section{Sensory and Motor Signs}

Among twenty six patients diagnosed with peripheral neuropathy, abnormalities in pin sensation was seen in all patients where as vibration sense was impaired in 10 $(38.46 \%)$ patients. Motor weakness was found in 5 (19.23\%) patients.

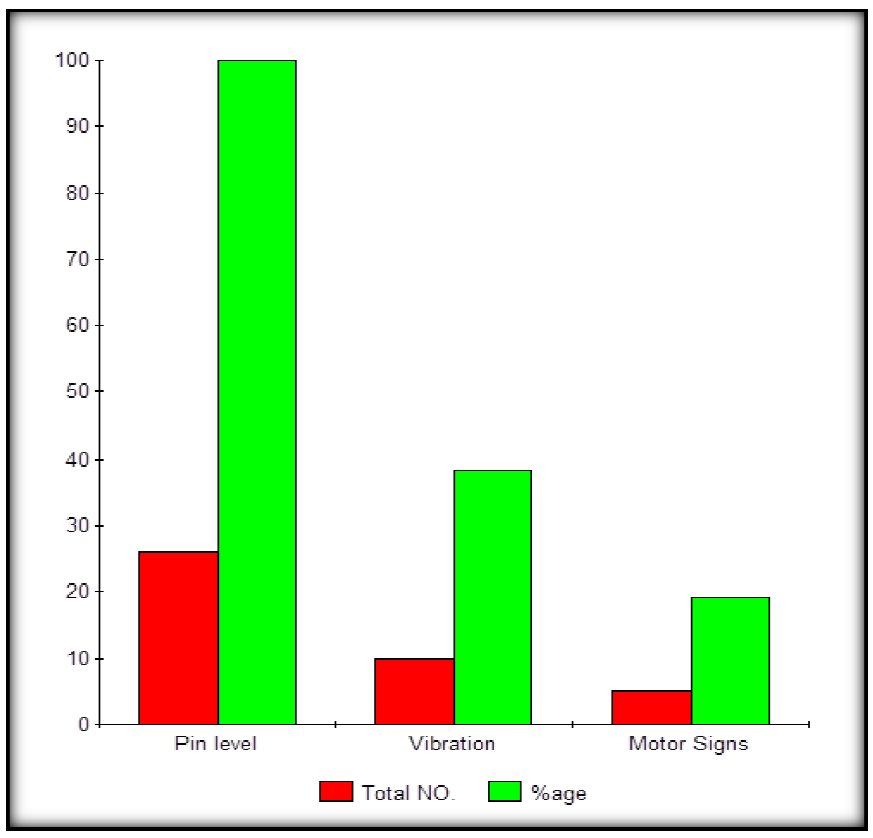

Figure 8: Distribution of patients according to motor and sensory signs.

\section{Grade of Neuropathy}

Twenty patients $(76.92 \%)$ had mild grade of neuropathy whereas six patients $(23.07 \%)$ had moderate grade of neuropathy. None of the patients had severe grade of neuropathy.

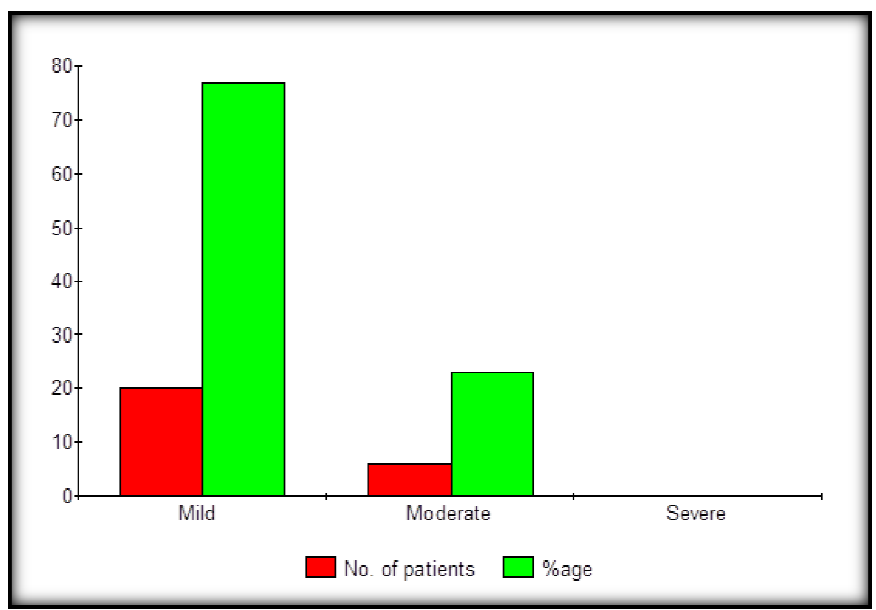

Figure 9: Distribution according to grade of neuropathy.

\section{Time of Onset of Neuropathy}

Colorectal carcinoma patients on FOLFOX chemotherapy were examined at baseline, after sixth cycle and after twelveth cycle. Most of patients $(n=21$ 80.76\%) had evidence of neuropathy after sixth cycle of chemotherapy 


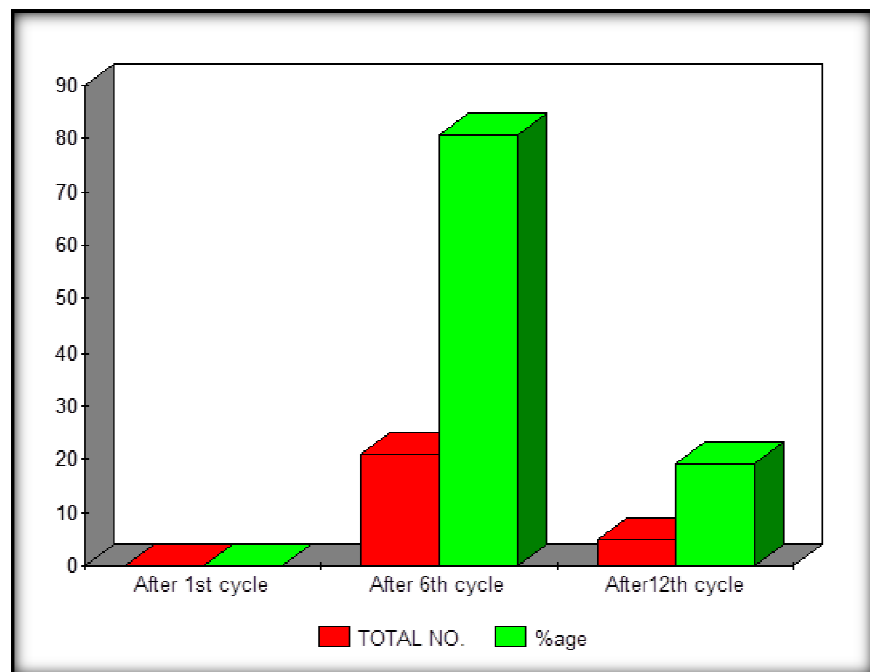

Figure 10: Distribution according to time of onset of neuropathy.

\section{Other Side Effects}

Of the forty six patients of colorectal cancer of FOLFOX4 chemotherapy neutropenia was seen in $43.47 \%(n=20)$ patients, nausea and vomiting were observed in $4.34 \%(n=2)$ of patients and diarrhea was there in $17.39 \%(\mathrm{n}=8)$ of patients.

\section{Discussion}

The present study was undertaken to determine the prevalence and characteristics of peripheral neuropathy in colorectal carcinoma patients on FO FOLFOX 4 therapy.

Peripheral neuropathy was observed in a significant number of patients $(n=26 ; 56.52 \%)$ on FOLFOX 4 therapy. Sensory neuropathy was found in $21(80.76 \%)$ patients and mixed pattern was seen in $05(19.23 \%)$ patients. The most frequent neurological adverse effect reported was parasthesias $(n=18$; $69.34 \%$ ) where as least frequently reported adverse event was neuropathic pain $(n=1 ; 3.84 \%)$. Females patients were more prone to risk of peripheral neuropathy $(57.69 \%)$ than male $(42.30 \%)$ patients.

A prospective study was done by Argyriou et al to determine the incidence and severity of oxaliplatin-induced peripheral neuropathy (OXLIPN). Evidence of OXLIPN was disclosed in 16 of the 25 patients (64\%). The results of this study were comparable to our results. ${ }^{[8]}$

In a descriptive study done by Vatandoust $S$ et al to determine persistent oxaliplatin induced peripheral neuropathy in colorectal carcinoma patients evidence of neuropathy was found in 25 (93\%) of 27 patients. This study revealed a very high prevalence of neuropathy as compared to our study. ${ }^{[9]}$

A descriptive study was done by Tofthagen $\mathrm{C}$ et al to explore the prevalence of neuropathic symptoms in patients with colorectal cancer receiving oxaliplatin as well as to explore symptom severity, distress, frequency, and neuropathic interference with activities. Cold sensitivity, tingling in the hands, and numbness in the hands were the most prevalent neuropathic symptoms, and cold sensitivity, nerve pain, and trouble with balance were the most severe symptoms. In our study also paresthesias (tingling) and numbness were the most prevalent symptoms. ${ }^{[10]}$

In a study done by Leonard et al. to see oxaliplatinassociated neurotoxicity using an interview-based questionnaire in patients with metastatic colorectal cancer, grade 1/2/3/4 dysesthesias and paresthesias were seen in $71 / 12 / 5 / 0$ and $66 / 20 / 7 / 1$ percent of patients. This correlates with our study as in our study parasthesias were found in $69.23 \%$ patients. ${ }^{[1]}$

A study done by Lin et al to identify early predictors of peripheral neuropathy and investigate the feasibility of using nerve conduction studies (NCS) showed that of 12 patients with neuropathy three had CTC grade 3 neuropathy, five had grade 2, and four had grade 1 neuropathy. In our study mild grade neuropathy was found in twenty patients and moderate grade in six patients. ${ }^{[12]}$

In a small, 25-patient prospective study specifically evaluating the development of OXIN, $56 \%$ of patients developed grade 1-2 OXIN and $8 \%$ developed grade 3 OXIN based on a modified TNS scoring. ${ }^{[13]}$ In the MOSAIC trial, $44 \%$ of patients had grade 2 or grade 3 neurotoxicity at the end of treatment. ${ }^{[14]}$ In our study mild grade of neuropathy was found in $76.92 \%$ and moderate grade was found in $23.07 \%$.

A study done by Park and colleagues to study the natural history of oxaliplatin-induced neuropathy in a population of 108 oxaliplatin-treated patients showed that $79.2 \%$ of patients reported residual neuropathic symptoms. Our study lacks long term follow up of these patients. ${ }^{[15]}$

In a study done by Bano et al to determine the neurological adverse effects in patients of advanced colorectal carcinoma treated with different schedules of FOLFOX it was observed that the most frequent neurological adverse effect in patients on FOLFOX 4 is paresthesias. This correlates with our study as in our study also parestheisas were the most common neurological adverse effect observed. ${ }^{[16]}$

Hence the prevalence of neuropathy in patients with colorectal cancer on FOLFOX 4 therapy was $56.52 \%$, with predominantly sensory neuropathy. The limitation of our study include long term follow up to determine the natural course as well as lack of Nerve conduction velocity studies to rule out latent neuropathy

\section{Conclusion}

- The aim of this study was to study prevalence and characteristics of peripheral neuropathy in colorectal carcinoma patients on FOLFOX 4 therapy.

- Forty six patients with colorectal carcinoma on FOLFOX 4 chemotherapy were evaluated. Patients were scored using modified neuropathy score.

- Mean age of the patients included in the study was 48.97 years (range 14-76 years).

- Out of these 25 patients were male and 21 patients were female.

- Prevalence of neuropathy was found in 26 patients (56.52\%).

- Sensory symptoms were experienced more commonly than motor symptoms. Among sensory symptoms tingling was the commonest neuropathic symptom $(\mathrm{n}=18,69.23 \%)$, 
followed by numbness ( $\mathrm{n}=5,19.23 \%$ ). Neuropathic pain occurred in one patient. Among motor symptoms, symptoms related to hand dexterity were most commonly observed $(n=4,15.38 \%)$.

- Abnormalities in pin sensation were seen in all patients whereas vibration sense was impaired in $10(38.46 \%)$ patients. Motor weakness was found in 5 (19.23\%) patients.

- Sensory neuropathy was the predominant form of neuropathy $(80.76 \%)$.

- Most of the patients (76.92\%) had mild grade neuropathy.

- Most of patients ( $n=2180.76 \%)$ had evidence of neuropathy after sixth cycle of chemotherapy.

This study shows that patients with colorectal carcinoma on FOLOFX 4 therapy develop peripheral neuropathy. Based on modified neuropathy score the prevalence of neuropathy in our study was $56.52 \%$. Majority of the patients developed sensory neuropathy of mild grade. Follow up of these patients is needed to evaluate whether peripheral neuropathy resolves or progresses and whether those patients who do not have clinical abnormalities, develop them later.

\section{References}

1. Ferlay J, Shin HR, Bray F, Forman D, Mathers C, Parkin DM. Estimates of worldwide burden of cancer in 2008: GLOBOCAN 2008. Int J Cancer. 2010;127:2893-917.

2. Simpson D, Dunn C, Curran M, Goa KL. Oxaliplatin: a review of its use in combination therapy for advanced metastatic colorectal cancer. Drugs. 2003;63:2127-56.

3. de Gramont A, Figer A, Seymour M, Homerin M, Hmissi A, Cassidy J et al. Leucovorin and fluorouracil with or without oxaliplatin as firstline treatment in advanced colorectal cancer. J ClinOncol. 2000;18 :2938-47.

4. de Gramont A, Vignoud J, Tournigand C, Louvet C, André T, Varette C et al. Oxaliplatin with high-dose leucovorin and 5-fluorouracil 48-hour continuous infusion in pretreated metastatic colorectal cancer. Eur J
Cancer.1997 ;33:214-9. among patients with Lynch syndrome. Gastroenterology. 2009; 137:1621-7.

5. Rosenberg L, Palmer JR, Zauber AG, Warshauer ME, Stolley PD, Shapiro S. A hypothesis: nonsteroidal anti-inflammatory drugs reduce the incidence of large-bowel cancer. J Natl Cancer Inst. 1991;83:355-8.

6. Howe GR, Benito E, Castelleto R, Cornée J, Estève J, Gallagher RP etal.Dietary intake of fiber and decreased risk of cancers of the colon and rectum: evidence from the combined analysis of 13 case-control studies. J Natl Cancer Inst.1992;84:1887-96.

7. Antonacopoulou AG, Argyriou AA, Scopa CD, Kottorou A, Kominea A, Peroukides $S$ et al.Integrin beta-3 L33P: a new insight into the pathogenesis of chronic oxaliplatin-induced peripheral neuropathy? Eur J Neurol. $2010 ; 17$ :963-8.

8. Argyriou AA, Polychronopoulos P, Iconomou G, Chroni E, Kalofonos HP. A review on oxaliplatin-induced peripheral nerve damage. Cancer Treatment Reviews. 2008;368-77.

9. Vatandoust S, Joshi R, Pittman KB, Esterman A, Broadbridge V, Adams $\mathrm{J}$ et al.. A descriptive study of persistent oxaliplatin-induced peripheral neuropathy in patients with colorectal cancer. Support Care Cancer.2013.

10. Tofthagen C, McAllister RD, McMillan SC. Peripheral neuropathy in patients with colorectal cancer receiving oxaliplatin. Clin J OncolNurs. 2011;15:182-8.

11. Leonard GD, Wright MA, Quinn MG, Fioravanti S, Harold N, Schuler $B$ et al. Survey of oxaliplatin-associated neurotoxicity using an interview-based questionnaire in patients with metastatic colorectal cancer. BMC Cancer. 2005;5:116.

12. Lin AY, Kouzminova NB .Predictors of oxaliplatin-induced peripheral neuropathy in patients with advanced colorectal cancer (CRC)JClinOncol 29: 2011.

13. Land SR, Kopec JA, Cecchini RS, Ganz PA, Wieand HS, Colangelo LH et al. Neurotoxicity from oxaliplatin combined with weekly bolus fluorouracil and leucovorin as surgical adjuvant chemotherapy for stage II and III colon cancer: NSABP C-07. J ClinOncol. 2007;25:2205-11.

14. Cavaletti G, Bogliun G, Marzorati L, Zincone A, Piatti M, Colombo $\mathrm{N}$,et al. Grading of chemotherapy-induced peripheral neurotoxicity using the Total Neuropathy Scale.2003; Neurology;61:1297-300.

15. Park SB, Lin CS, Krishnan AV, Goldstein D, Friedlander ML, Kiernan MC. Long-term neuropathy after oxaliplatin treatment: challenging the dictum of reversibility. Oncologist. 2011;16:708-16.

16. Bano N, Najam R, Mateen A. Neurological Adverse Effects in Patients of Advanced Colorectal Carcinoma Treated with Different Schedules of FOLFOX.Chemotherapy Research and Practice. 2013 ;2013:1-7.

Copyright: (C) the author(s), 2019. It is an open-access article distributed under the terms of the Creative Commons Attribution License (CC BY 4.0), which permits authors to retain ownership of the copyright for their content, and allow anyone to download, reuse, reprint, modify, distribute and/or copy the content as long as the original authors and source are cited.

How to cite this article: Bharti A, Arora I, Chalotra V. Neuropathy in Carcinoma Colon Patients Prevelence and Characteristics of Neuropathy in Carcinoma Colon Patients on Folfox 4 Therapy. Acad. J. Surg. 2019;2(2):4-9.

DOI: dx.doi.org/10.21276/ajs.2019.2.2.2

Source of Support: Nil, Conflict of Interest: None declared. 